\title{
Reliability of computed tomography scans in the diagnosis of chronic rhinosinusitis
}

\author{
Marcin Frączek ${ }^{1, A-D, F}$, Marcin Masalski ${ }^{1, B}$, Maciej Guziński2 ${ }^{2, E}$ \\ 1 Department of Otolaryngology, Wroclaw Medical University, Poland \\ ${ }^{2}$ Department of Radiology, Wroclaw Medical University, Poland \\ A - research concept and design; $\mathrm{B}$ - collection and/or assembly of data; $\mathrm{C}$ - data analysis and interpretation; \\ $D$ - writing the article; $E$ - critical revision of the article; $F$ - final approval of the article
}

\section{Address for correspondence}

Marcin Frączek

E-mail: raucedo@wp.pl

Funding sources

None declared

Conflict of interest

None declared

Received on May 29, 2015

Reviewed on June 22, 2017

Accepted on November 28, 2017

\section{Abstract}

Background. Paranasal computed tomography (CT) has become the investigation method of choice to confirm or exclude the diagnosis of chronic rhinosinusitis (CRS) on the basis of its ability to deliver objective data regarding the presence of inflamed mucosa or polyps.

Objectives. The aim of the study was to assess the reliability of CT scan findings among untreated CRS patients without the presence of polyps in a nasal endoscopy.

Material and methods. Among patients with clinically demonstrated CRS considered for surgery, 93 subjects who had had 2 CT scans performed at different time points in the diagnostic process were enrolled into the study. Paranasal sinus involvement on both CT scans was scored using the Lund-Mackay (L-M) and modified Lund-Mackay scales. Both CT exams served to assess the extent of the potential endoscopic sinus surgery.

Results. The time interval between (T scans ranged from 31 to 1,162 days (mean: 338 days). The L-M scores from the ${ }^{\text {st }} \mathrm{CT}$ examination correlated statistically with the results of the $2^{\text {nd }} \mathrm{C}(\mathrm{r}=0.86 ; p<0.05)$. When compared to the $1{ }^{\text {st }}$ scan, the L-M score in the $2^{\text {nd }} \mathrm{CT}$ scan remained the same in 36 patients (39\%), increased in 23 patients (25\%) and decreased in 34 patients (36\%). There was no statistically significant correlation between the change in the L-M scores and the time interval between CT examinations.

Conclusions. The present study indicates that mucosal thickening within paranasal sinuses among untreated patients with CRS is stable over short- and middle-time intervals, regardless of the initial intensity of the disease. The time delay between the CT examination and qualification for surgery does not influence the decision regarding the performance of the operation. The results suggest the conclusion that repeating CT scans in symptomatic, untreated patients with CRS should be seriously considered.

Key words: surgery, diagnosis, reliability, tomography, sinusitis

DOI

10.17219/acem/80858

\section{Copyright}

Copyright by Author(s)

This is an article distributed under the terms of the

Creative Commons Attribution Non-Commercial License

(http://creativecommons.org/licenses/by-nc-nd/4.0/) 


\section{Introduction}

For many years, chronic rhinosinusitis (CRS) was commonly identified solely on the basis on the patient's subjective symptoms. ${ }^{1}$ Later, in order to confirm a diagnosis, the symptom-based definition was reevaluated by the use of either radiological imaging or nasal endoscopy. ${ }^{2}$ Computerized tomography $(\mathrm{CT})$ provided detailed images of the sinuses and gave the examiner a clear view of those areas that are of key importance in the pathogenesis of CRS. ${ }^{3}$ Thus, due to the relatively unsatisfactory specificity and positive predictive value of symptom-based diagnostic criteria, many physicians rely extensively on CT findings. ${ }^{4}$ It is often the case among patients with mild disease severity or isolated symptoms, but also among those with CRS without polyps when the doctor lacks the confidence to treat without further imaging.

The growing availability of CT scanners means that such an examination is requested frequently, often at very distinct stages of the diagnostic or therapeutic process. Increasingly easy access and the promotion of low-dose devices may further encourage the performance of subsequent examinations. As a result of such a tendency, doctors might encounter new patients with already performed $\mathrm{CT}$, and must decide whether to repeat the scan or to rely on the old one. The question becomes particularly important when surgical intervention is being considered. At the same time, data regarding the durability of CT scan findings, especially in respect to the severity of CRS, is still rare, and the issue is practically not discussed and questioned in the literature. ${ }^{5}$

Besides extra costs, repeated paranasal CT examinations are associated with additional radiation exposure, which is principally important in respect to the eye lens and thyroid gland. ${ }^{6}$ The dose of radiation related to paranasal CT study is comparable to natural background radiation for about 8 months. ${ }^{7}$ This is the same length of time the human body needs to repair the radiation injury caused by such an examination. Furthermore, the overall lower mean age of CRS patients and the fact that repeated $\mathrm{CT}$ examinations are performed for benign disease should raise serious concerns.

The aim of the present paper was to assess the reliability of CT scan findings among untreated CRS patients without polyps apparent in nasal endoscopy. Additionally, the evaluation of distinct paranasal CT scans performed in the same untreated patients allowed an analysis of the natural history of the condition.

\section{Material and methods}

Patients with CRS consulting or admitted to the Department of Otolaryngology at Wroclaw Medical University for surgery were considered to be enrolled into the study. Conditions for inclusion were a lack of polyps in nasal endoscopy and a previously performed CT scan. The $1^{\text {st }} \mathrm{CT}$ examination was conducted at the request of otolaryngologists from the outpatient department, or general practitioners. In the study group there were no patients with bronchial asthma or allergic rhinitis. Symptomatic patients for whom thorough conservative management had not been successful and who still met the diagnostic criteria for CRS were qualified for functional endoscopic sinus surgery (ESS).

The $2^{\text {nd }} \mathrm{CT}$ scan was considered after admission only in the following clinical situations: when the time interval between scans was longer than 6 months; when the $1^{\text {st }} \mathrm{CT}$ was not appropriate for an image guided system if required; in the case of problems with the examination on CD-ROM or when symptoms clearly differed from the intensity of changes on the $1^{\text {st }} \mathrm{CT}$ scan. The $\mathrm{CT}$ examination was performed on a GE Discovery $750 \mathrm{HD}$ scanner (General Electric Healthcare, Milwaukee, USA) using the low-dose protocol: tube potential $120 \mathrm{kVp}, 45 \mathrm{mAs}$; detector configuration $64 \times 0.625 \mathrm{~mm}$; pitch 1.3 ; section thickness 0.625 ; and gantry rotation time $0.4 \mathrm{~s}$. Patients were scanned with a scanning range from the top of the frontal sinuses to the level of hard palate and from the tip of the nose to the region posterior to the clivus. The images were reconstructed with an adaptive statistical iterative reconstruction (ASIR) algorithm using 50\% ASIR.

Only symptomatic patients who had not been treated pharmacologically between CT studies were finally accepted. Each patient gave written consent for the CT examination to be performed and to participate in the study. The study protocol was approved by our institutional review board.

\section{Computed tomography scoring systems}

In each case, both paranasal sinus CT scans were reviewed in random order and scored according to 2 staging systems: the Lund-Mackay (L-M) and the modified L-M. CT scans were subjected to an independent double-blind review by 2 physicians, and the results were compared.

\section{Lund-Mackay scoring system}

The L-M staging system is a measure of the degree of opacification in the paranasal sinuses. ${ }^{8,9}$ When inflammation occupied $0 \%$ of the CT image, a score of 0 was assigned; a score of 2 was assigned when the changes occupied 100\% of the sinus. All other degrees of inflammation were scored as 1 . For the ostiomeatal complex $(\mathrm{OMC}): 0$ = not occluded; $2=$ occluded. The total score might range from 0 to 24 .

\section{Modified Lund-Mackay scoring system}

It classifies the volume of inflammation in each sinus into 4 strata using intervals of $33 \% .^{10}$ For the sinuses with no inflammation, a score of 0 was assigned; a score of 1 for inflammation occupying 1-33\%; a score of 2 for inflammation in $34-66 \%$ of the sinus; a score of 3 for sinuses 
occupied in 67-99\%; and a score of 4 in the cases with total sinus opacification. For the OMC: $0=$ not occluded; 2 = occluded. The total score might range from 0 to 44 .

Patients were excluded if any of the elements of the scoring systems could not be properly evaluated.

\section{Extent of the potential endoscopic sinus surgery according to $\mathrm{CT}$ examinations}

The volume of sinus opacification determines the extent of the endoscopic surgery. However, a small change in the L-M score resulting from the time flow may have no effect on the extent of operation. Additionally, patients with equal L-M scores may require surgery of different extents.

In order to clarify the above ambiguities, the $1^{\text {st }}$ and $2^{\text {nd }} \mathrm{CT}$ examinations served to estimate the range of potential ESS in patients with L-M scores $\geq 1$. The surgery score was evaluated according to the modified classification proposed by Lund and Mackay, which included: uncinectomy, middle meal antrostomy, anterior and posterior ethmoidectomy, sphenoidectomy, and frontal recess surgery $(0=$ no procedure; $1=$ procedure done $) .{ }^{11}$ Total scores range from 0 to 12 .

\section{Statistics}

Statistical analysis was performed with STATISTICA software v. 12 (StatSoft, Tulsa, USA). The correlation between the extent of disease on the $1^{\text {st }}$ and $2^{\text {nd }} \mathrm{CT}$ scan assessed by the L-M and modified L-M scales was effected using Pearson's r correlation analysis. Spearman's rho was used to measure the correlation between ranked variables. Statistical significance of correlation coefficients $r$ and rho was tested with the t-test. All tests were verified at the significance level $\alpha=0.05$. A p-value $<0.05$ was considered statistically significant.

\section{Results}

A total of 93 adult patients met the inclusion criteria and were enrolled into the study. There were 41 (44\%) female and $52(56 \%)$ male patients. The average patient age at the time of the $1^{\text {st }}$ examination was 43.7 years (18-76 years). The time interval between CT scans ranged from 31 to 1,162 days (mean: 338 days; SD: 238 days).

The L-M scores ranged from 1 to 16 (mean: 7.48; SD: 4.26) in the $1^{\text {st }} \mathrm{CT}$ scan and from 0 to 18 (mean: 7.43; SD: 4.57) in the $2^{\text {nd }} \mathrm{CT}$ examination (Fig. 1). The modified L-M scores ranged from 1 to 34 (mean: 11.33; SD: 8.20 ) in the $1^{\text {st }} \mathrm{CT}$ examination and from 0 to 36 (mean: 11.45 ; SD: 8.29) in the $2^{\text {nd }} \mathrm{CT}$ scan.

Changes in L-M and modified L-M scores between CT scans were not statistically correlated with the patients' sex and age.

\section{The correlation between the scores from the $1^{\text {st }}$ and $2^{\text {nd }}$ CT scans}

The severity of sinus involvement on the $1^{\text {st }} \mathrm{CT}$ scan correlated statistically with sinus opacification on the $2^{\text {nd }}$ scan as scored by the L-M system $(r=0.86 ; \mathrm{p}<0.05)$ (Fig. 2). Similarly, a statistically significant association was found for the modified L-M scale ( $\mathrm{r}=0.83$; $\mathrm{p}<0.05)$. The comparison disclosed that both scales are reliable, but L-M insignificantly more so.

\section{Does the change in L-M score correlate with the change in modified L-M score?}

The difference in sinus opacification between the $1^{\text {st }}$ and $2^{\text {nd }} \mathrm{CT}$ scans assessed by the L-M scale correlated significantly with the same change scored by the modified L-M system (Spearman's rho $=0.87 ; \mathrm{p}<0.001$ ).

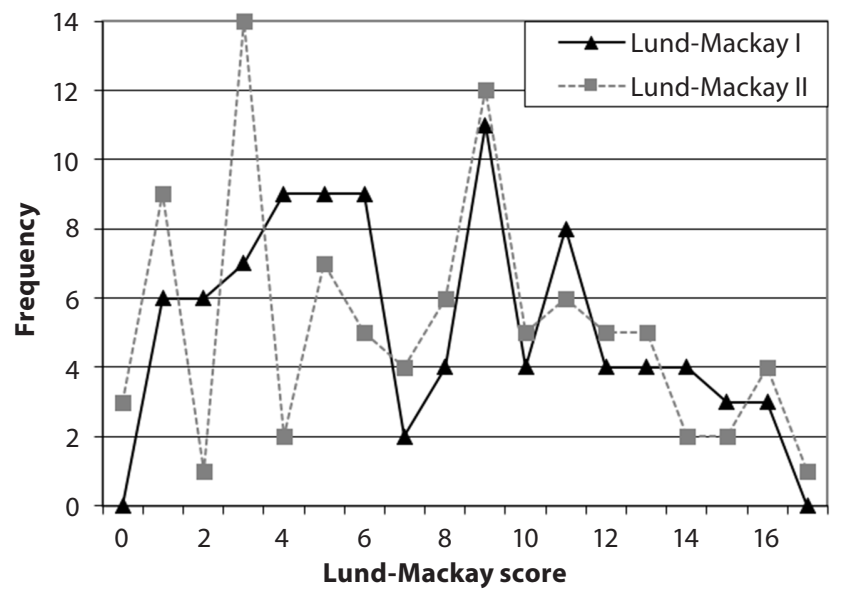

Fig. 1. The distribution of Lund-Mackay scores for the $1^{\text {st }}$ and the $2^{\text {nd }}$ computed tomography scans

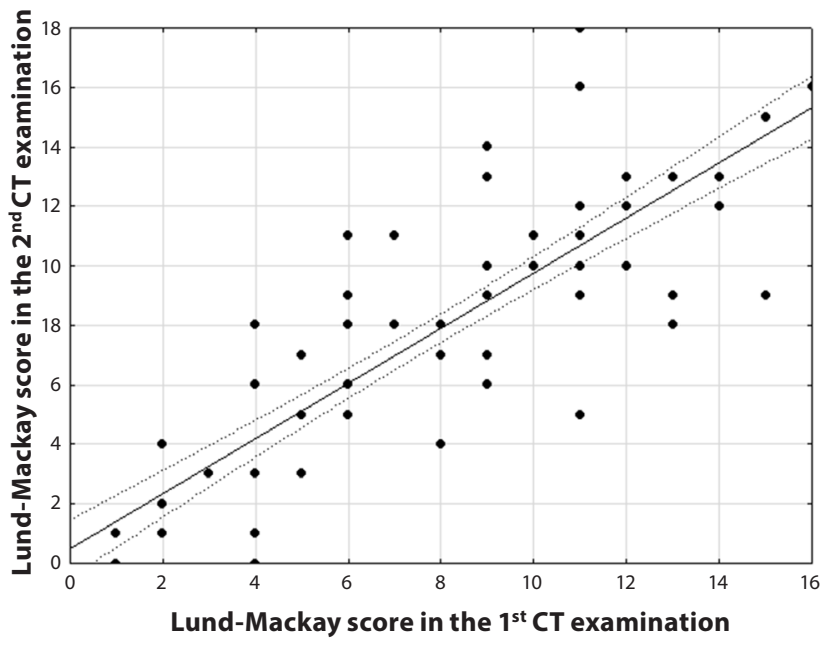

Fig. 2. Scatter-plot of the correlation between the $1^{\text {st }}$ and the $2^{\text {nd }}$ computed tomography scans 


\section{Change in L-M score between scans as a function of time interval between scans}

The L-M score for 36 (39\%) patients remained the same over time, increased in 23 (25\%) and decreased in 34 (36\%) cases. There was no statistically significant correlation between the change in the L-M scores and the time intervals between the $1^{\text {st }}$ and $2^{\text {nd }} \mathrm{CT}$ examination (Fig. 3, 4).

In addition, patients were divided into 2 groups depending on the length of time intervals between CT examinations - above and below 1 year. In patients with a longer interval between scans, the change in the L-M scores was more pronounced, although the difference was not statistically significant.

\section{Does the initial sinus opacification correlate with subsequent changes in mucosal inflammation?}

There was no correlation between the initial sinus opacification and the subsequent change in inflammation intensity measured by the L-M and modified L-M systems. The initial severity of inflammation within the sinuses cannot determine how epithelial changes will evolve over time. There was no difference in the change in inflammation severity among patients with low-stage ( $\mathrm{L}-\mathrm{M} \leq 3$; $\mathrm{n}=19)$ and high-stage CRS (L-M > 3; $\mathrm{n}=74)$.

\section{Does the change in L-M score between scans influence the extent of potential sinus surgery?}

The mean extent of potential endoscopic sinus surgery assessed by modified L-M classification on the basis of the $1^{\text {st }}$ and $2^{\text {nd }}$ CT examination was 6 (SD: 3.22 ) and 5.96 (SD: 3.31), respectively. Two (2\%) patients with $\mathrm{L}-\mathrm{M}=1$ did not qualify for surgery on the basis of the $1^{\text {st }} \mathrm{CT}$ scan. According to the $2^{\text {nd }}$ CT scan, surgical treatment was cancelled in 8 (8.6\%) patients including: the 2 mentioned above with unchanged $\mathrm{L}-\mathrm{M}=1,3$ consecutive patients who had no inflammatory changes in CT $(\mathrm{L}-\mathrm{M}=0)$ and 3 patients with the L-M score decreased to 1 . The scope of surgery based on the $2^{\text {nd }} \mathrm{CT}$ did not change compared to that performed on the basis of the $1^{\text {st }} \mathrm{CT}$ in 45 patients (48\%), while it increased in 18 (19\%) and in 30 (32\%) decreased.

Changes in sinus opacification between $1^{\text {st }}$ and $2^{\text {nd }} \mathrm{CT}$ examinations correlated significantly with a change in the extent of potential sinus surgery (Spearman's rho $=0.73 ; \mathrm{p}<0.001$ for L-M, and Spearman's rho $=0.68 ; \mathrm{p}<0.001$ for modified L-M scale).

\section{Discussion}

The diagnosis and management of chronic rhinosinusitis still continue to provide many difficulties. One of the current questions is how stable over time is mucosal thickening

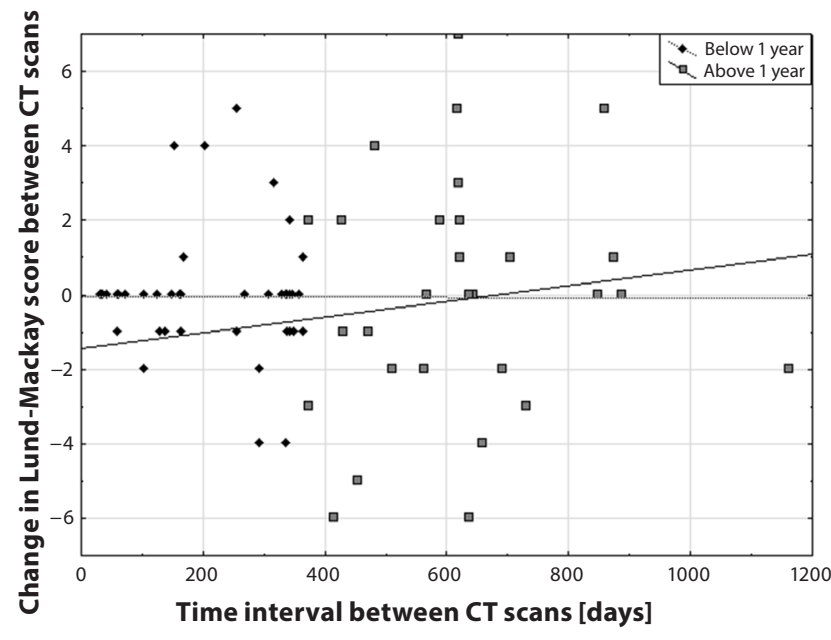

Fig. 3. Change in Lund-Mackay scores between scans as a function of time intervals between scans

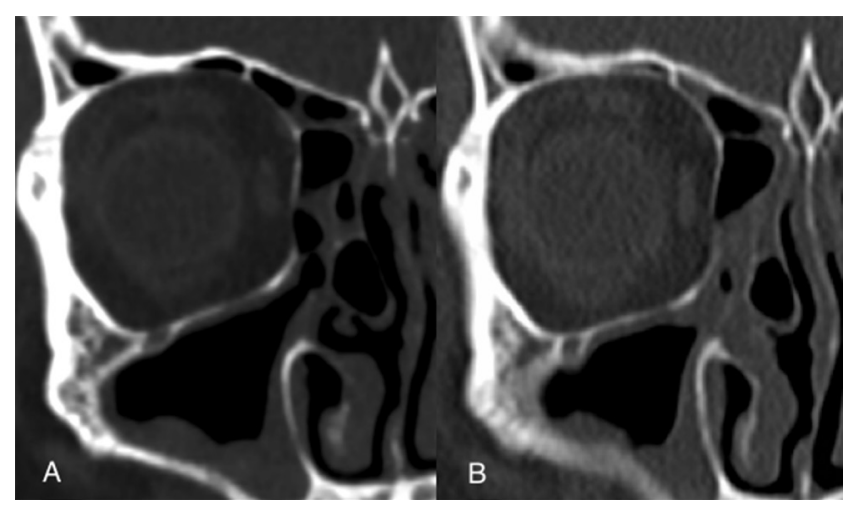

Fig. 4. Coronal images of CT scans obtained in untreated patient with chronic rhinosinusitis in 1-year interval; slight change in sinus opacification between the $1^{\text {st }}(A)$ and the $2^{\text {nd }}(B) C T$ examination

within sinuses. Our results allow us to state that the CT scan findings in untreated CRS without polyps generally remain at the same level over short- and middle-time intervals. Contrary to the common expectation, mucosal thickening was not demonstrated to have a clear tendency to grow over time. Inflammatory changes may either slightly increase or decrease, and the direction in which this will evolve is unpredictable. Herein, the chance for a larger change grew insignificantly with the length of the follow-up. Second CT scans performed after 12 months or longer revealed more pronounced mucosal thickening compared to the scans repeated after a shorter time. Such observations also mean that mucosal disease among CRS subjects increases over a longer period than that considered in this study.

It was proved herein that CT scans in CRS possess good test-retest reproducibility. In contrast to Bhattacharyya, who analyzed a similar issue, we included only subjects without polyps in nasal endoscopy. ${ }^{5}$ The essential advantage of the present study is the higher number of patients and a much longer follow-up period. 
These results allow us to conclude that repeating CT scans in symptomatic, untreated patients with CRS should be seriously considered. Spontaneous withdrawal of the mucosal thickening to the extent that disqualifies patients from surgery is very unlikely. One of the goals of pre-operative $\mathrm{CT}$ is to delineate the extent of disease to omit unnecessary intervention in disease-free sinuses. The outcomes of the study indicate that the time delay between CT examination and qualification for surgery should, however, not influence decisions regarding the surgery, but it might slightly change its extent.

In the adult population, a quantified L-M scale can be used to distinguish between patients with and without CRS. ${ }^{12}$ Typically, L-M scores $>3$ are highly likely to represent true CRS. L-M scores $\leq 3$, categorized as low-stage CRS, are unclear, and additional clinical judgment or corroborating data are required to establish diagnoses. Although recalcitrant, low-stage CRS forms a relatively small subset, patients with minimal mucosal disease on CT scans but having symptoms suggestive of CRS can cause some therapeutic dilemmas. In these patients in particular, the fact that the clinical course of CRS is characterized by variations in severity should be taken into consideration. It seems to be also possible that CT can identify "incidental" mucosal thickening, which does not represent true disease. ${ }^{13}$ Such incidental opacification of sinuses on CT scans is identified in $27-45 \%$ of asymptomatic individuals. ${ }^{14}$ However, according to the same data, as much as $40 \%$ of patients who met the criteria for the diagnosis of CRS do not have radiological evidence of disease. ${ }^{15,16}$ Thus, among subjects with minimally affected CT scans, despite the failure of medical therapy, there is often a reluctance to perform surgery. Repeating CT examinations might hypothetically guard against subjecting healthy patients to invasive procedures or extended therapy. In the present study, changes in sinus opacification over time among patients with low-stage CRS did not differ significantly from those in patients with high-stage CRS. The results indicate that even a limited mucosal disease is stable over time. This supports the current approach that the presence of a minimally affected CT scan should not exclude the diagnosis of CRS. ${ }^{17}$ Moreover, the results encourage considering the implementation of ESS among symptomatic low-stage CRS patients resistant to medical management.

The major drawback of the Lund-Mackay system is its inability to subgrade the volume of inflammatory disease in sinuses. ${ }^{10}$ For this reason, the new staging system was proposed by 5 American societies, called the modified L-M scale. ${ }^{10}$ Although the scale has a more efficient ability to subgrade the level of inflammation, the application of modified L-M in the present study did not give any benefits. Furthermore, the L-M scoring system showed a higher level of reliability, which is consistent with earlier data published by Okushi et al. ${ }^{18}$

Despite the propagation of the symptom-based diagnosis of CRS and the good specificity of endoscopic examination, in many situations physicians are willing to repeat $\mathrm{CT}$, thus forgetting the principle of radiation protection: as low as reasonably achievable (ALARA). This raises the danger that CT will be over-prescribed as a diagnostic tool in CRS. The results of the present study indicate that inflammatory changes within paranasal sinuses in symptomatic, untreated patients with CRS are stable over time, regardless of the initial intensity. Thus, in those cases when complaints persist, repeating CT scans for reasons other than the planning of surgical intervention is not justified. Such a conclusion is also important in terms of the increasing public awareness of the dose burden related to that examination.

\section{References}

1. Hadley JA, Schaefer SD. Clinical evaluation of rhinosinusitis: History and physical examination. Otolaryngol Head Neck Surg. 1997;117:S8-11.

2. Fokkens WJ, Lund VJ, Mullol J, et al. European position paper on rhinosinusitis and nasal polyps 2012. Rhinol Suppl. 2012;3:1-298.

3. Bhattacharyya N, Lee LN. Evaluating the diagnosis of chronic rhinosinusitis based on clinical guidelines and endoscopy. Otolaryngol Head Neck Surg. 2010;143:147-151.

4. White PS, Maclennan AC, Connolly AA, Crowther J, Bingham BJ. Analysis of CT scanning referrals for chronic rhinosinusitis. J Laryngol Otol. 1996;110:641-643.

5. Bhattacharyya N. Test-retest reliability of computed tomography in the assessment of chronic rhinosinusitis. Laryngoscope. 1999;109:1055-1058.

6. Mazonakis M, Tzedakis A, Damilakis J, Gourtsoyiannis N. Thyroid dose from common head and neck CT examinations in children: Is there an excess risk for thyroid cancer induction? Eur Radiol. 2007;17:1352-1357.

7. Ibrahim M, Parmar H, Christodoulou E, Mukherji S. Raise the bar and lower the dose: Current and future strategies for radiation dose reduction in head and neck imaging. AJNR Am J Neuroradiol. 2014;35:619-624.

8. Lund VJ, Kennedy DW. Quantification for staging sinusitis: The staging and therapy group. Ann Otol Rhinol Laryngol Supl. 1995;167:17-21.

9. Hopkins C, Browne JP, Slack R, Lund V, Brown P. The Lund-Mackay staging system for chronic rhinosinusitis: How is it used and what does it predict? Otolaryngol Head Neck Surg. 2007;137:555-561.

10. Meltzer EO, Hamilos DL, Hadley JA, et al. Rhinosinusitis: Establishing definitions for clinical research and patient care. J Allergy Clin Immunol. 2004;114:155-212.

11. Lund VJ, Kennedy DW. Staging for rhinosinusitis. Otolaryngol Head Neck Surg. 1997;117:S35-S40.

12. Bhattacharyya N, Fried MP. The accuracy of computer tomography in the diagnosis of chronic sinusitis. Laryngoscope. 2003;113:125-129.

13. Bhattacharyya N. Do maxillary sinus retention cysts reflect obstructive sinus phenomena? Arch Otolaryngol Head Neck Surg. 2000;126:1369-1371.

14. Calhoun K, Waggenspack G. CT evaluation of the paranasal sinuses in symptomatic and asymptomatic populations. Otolaryngol Head Neck Surg. 1991;104:480-483.

15. Stankiewicz JA, Chow JM. A diagnostic dilemma for chronic rhinosinusitis: Definition, accuracy and validity. Am J Rhinol. 2002;16:199-202.

16. Ferguson BJ, Narita M, Yu VL, Wagener MM, Gwaltney JM Jr. Prospective observational study of chronic rhinosinusitis: Environmental triggers and antibiotic implications. Clin Infect Dis. 2012;54:62-68.

17. Kenny TJ, Duncavage J, Bracikowski J, Yildirim A, Murray JJ, Tanner SB. Prospective analysis of sinus symptoms and correlation with paranasal computed tomography scan. Otolaryngol Head Neck Surg. 2001;125:40-43.

18. Okushi T, Nakayama T, Morimoto S, et al. A modified Lund-Mackay system for radiological evaluation of chronic rhinosinusitis. Auris Nasus Larynx. 2013;40:548-553. 